ка сигналов : пер. с англ. М. : Связь, 1979. 416 с.

5. Rundell W., Sacks P. E. Reconstruction techniques for classical inverse Sturm - Liouville problems // Mathematics of Computation. 1992. Vol. 58, № 197. P. 161-183. DOI: 10.1090/S0025-5718-1992-1106979-0.

6. Freiling G., Yurko V. A. Inverse Sturm-Liouville Problems and Their Applications. Huntington ; N. Y. : NOVA Science Publ., 2001. 305 p.

7. Onпенгеймер A. В., Шафер Р. В. Цифровая обработ-
8. Винокуров B. A., Садовничий B. А. Асимптотика любого порядка собственных значений и собственных функций краевой задачи Штурма - Лиувилля на отрезке с суммируемым потенциалом // Изв. РАН. Сер. математическая. 2000. Т. 64, № 4. C. 47-108. DOI: $10.4213 / \operatorname{im} 295$

\title{
Numerical Solution of Inverse Spectral Problems for Sturm - Liouville Operators with Discontinuous Potentials
}

\begin{abstract}
L. S. Efremova
Saratov State University, 83, Astrakhanskaya str., Saratov, 410012, Russia, liubov.efremova @ gmail.com

We consider Sturm - Liouville differential operator with potential having a finite number of simple discontinuities. This paper is devoted to the numerical solution of such inverse spectral problems. The main result of this work is a procedure that is able to recover both the points of discontinuities as well as the heights of the jumps. Following, using these results, we may apply a suitable numerical method (for example, the generalized Rundell -Sacks algorithm with a special form of the reference potential) to reconstruct the potential more precisely.
\end{abstract}

Key words: Sturm - Liouville differential operator, inverse spectral problem, discontinuous potential, numerical solution.

This work was supported by the Russian Foundation for Basic Research (project no. 14-01-31042).

\section{References}

1. Levitan B. M. Inverse Sturm-Liouville Problems. Utrecht, VNU Sci. Press, 1987, 240 p.

2. Marchenko V. A. Sturm-Liouville operators and applications. Basel, Birkhäuser, 1986. 367 p.

3. Ignatiev M. Yu., Yurko V. A. Numerical methods for solving inverse Sturm - Liouville problems. Results in Math., 2008, vol. 52, pp. 63-74. DOI: 10.1007/s00025007-0276-y.

4. Rafler M., Böckmann C. Reconstruction method for inverse Sturm-Liouville problems with discontinuous potentials. Inverse Problems, 2007, vol. 23, no. 3, pp. 933-946. DOI: 10.1088/0266-5611/23/3/006.

5. Rundell W., Sacks P. E. Reconstruction techniques for classical inverse Sturm-Liouville problems. Mathema- tics of Computation, 1992, vol. 58, no. 197, pp. 161-183. DOI: 10.1090/S0025-5718-1992-1106979-0.

6. Freiling G., Yurko V. A. Inverse Sturm-Liouville Problems and Their Applications. Huntington, New York, NOVA Science Publ., 2001, 305 p.

7. Oppenheim A. V., Schafer R. W. Discrete-time Signal Processing. Prentice-Hall, 1975, 585 p.

8. Vinokurov V. A., Sadovnichii V. A. Asymptotics of any order for the eigenvalues and eigenfunctions of the Sturm-Liouville boundary-value problem on a segment with a summable potential. Izvestiya : Mathematics, 2000, vol. 64, iss. 4, pp. 695-754. DOI: http://dx.doi.org/10.4213/im295.

УДК 517.51

\section{ОБ ОПЕРАТОРЕ ДИФФЕРЕНЦИРОВАНИЯ НА КОМПАКТНЫХ НУЛЬ-МЕРНЫХ ГРУППАХ}

\section{Ю. С. Кpycc}

Аспирант кафредры математического анализа, Саратовский государственный университет им. Н. Г. Чернышевского, KrussUS@gmail.com

Для одномерного случая указаны условия, при которых оператор дифрференцирования не зависит от ортонормированной системы, с помощью которой определен. Для многомерного случая указаны условия, при которых оператор дисрференцирования не зависит от способа преобразования многомерной компактной нуль-мерной группы в одномерную. Получен явный вид аннуляторов в многомерной компактной нуль-мерной группе.

Ключевые слова: нуль-мерные группы, псевдодиффреренциальный оператор, сильная Р-ичная производная, сильный P-ичный интеграл. 


\section{ВВЕДЕНИЕ}

P. L. Butzer и H. J. Wagner [1] ввели понятие сильной двоичной производной $D(f)$ для функций, интегрируемых на двоичной группе Кантора. Б. И. Голубов [2] обобщил это определение на случай функций, интегрируемых на $\mathbb{R}^{+}$или (что то же самое) на локально компактной двоичной группе, и определил как производную, так и обобщенный сильный интеграл. С. С. Волосивец [3] определил на $\mathbb{R}^{+}$модифицированную сильную $\mathbf{P}$-ичную производную и модифицированный сильный $\mathbf{P}$-ичный интеграл.

Понятия модифицированной сильной $\mathbf{P}$-ичной производной и модифицированного сильного $\mathbf{P}$-ичного интеграла тесно связаны с понятием псевдодифференциального оператора $D_{\varphi}(f, x)=(\varphi(\chi) \hat{f}(\chi))^{\vee}(x)$, определяемого символом $\varphi$. Выбирая подходящим образом символ $\varphi$, можно получить как модифицированную сильную $\mathbf{P}$-ичную производную, так и модифицированный сильный $\mathbf{P}$-ичный интеграл. На этом пути сразу получаем, что композиция операторов дифференцирования и интегрирования в любом порядке дает тождественный оператор. На компактной группе оператор дифференцирования умножает ряд Фурье по системе характеров дифференцируемой функции на некоторую последовательность (символ). В 2002 г. С. В. Козырев обнаружил, что система Хаара представляет собой собственные функции псевдодифференциального оператора, определенного в поле $p$-адических чисел [4]. Поэтому возникает вопрос: как действует оператор дифференцирования на ряд Фурье - Хаара? В предлагаемой работе найдены условия на символ, при которых результат дифференцирования не зависит от выбранной нами системы (теорема 3).

В многомерном случае стоит несколько иная задача. Многомерную группу можно преобразовать в одномерную разными способами [5-6], получая при этом различные основные цепочки подгрупп. В связи с чем возникает вопрос: при каких условиях оператор дифференцирования не зависит от того, какой способ преобразования многомерной группы в одномерную мы используем? Ответ на этот вопрос дается в теореме 5. Для доказательства этой теоремы найдено явное представление аннуляторов основной цепочки произведения групп $\mathbb{G}$ (теорема 4$)$.

\section{1. ОСНОВНЫЕ ОПРЕДЕЛЕНИЯ}

Пусть $(G, \dot{+})$ - компактная аддитивная топологическая группа, топология в которой задана счетной системой вложенных подгрупп:

$$
G=G_{0} \supset G_{1} \supset G_{2} \supset \ldots \supset G_{n} \supset \ldots
$$

таких, что $\bigcap_{n=0}^{\infty} G_{n}=\{0\}(0-$ нулевой элемент группы $G)$. Таким образом, базу топологии в $G$ образуют смежные классы $\left(G_{n} \dot{+} h\right)\left(n \in \mathbb{N}_{0}=\mathbb{N} \cup\{0\}, h \in G\right)$, из которых любые два либо не пересекаются, либо один включается в другой. Такую группу называют нуль-мерной. Из компактности $G$ следует, что все фактор-группы $G_{n} \backslash G_{n+1}$ конечны. Обозначим через $p_{n}$ порядок такой фактор-группы. Можно считать, что все $p_{n}$ - простые числа, так как, в противном случае, используя теорему Силова, можно уплотнить цепочку подгрупп так, что порядки фактор-групп $G_{n} \backslash G_{n+1}$ станут простыми числами. Обозначим $m_{0}=1, m_{n+1}=m_{n} p_{n}$. Тогда при каждом $n \in \mathbb{N}$ группа $G$ есть дизъюнктное объединение $m_{n}$ смежных классов $G_{n} \dot{+} h_{i},\left(i=\overline{0, m_{n}-1}\right)$. Все смежные классы в объединении с пустым множеством образуют полукольцо $K$. На $K$ можно ввести меру $\mu$ равенством $\mu\left(G_{n} \dot{+} h_{i}\right)=\mu G_{n}=$ $=1 / m_{n}$. После этого меру $\mu$ можно продолжить на $\sigma$-алгебру $\mu^{*}$ измеримых множеств, например, по схеме Каратеодори. Получим меру, инвариантную относительно сдвига. На борелевских множествах она совпадает с мерой Хаара.

На $G$ можно определить интеграл по схеме Лебега, который инвариантен относительно сдвига и является абсолютно сходящимся. Характеры группы $G$ будем обозначать через $\chi$, а группу всех характеров - через $X$. Совокупность характеров $\chi$ таких, что $\chi(x)=1$ для любого $x \in G_{n}$, называют аннулятором группы $G_{n}$ и обозачают $G_{n}^{\perp}$. Аннуляторы образуют возрастающую цепочку подгрупп группы $X$ :

$$
\{1\}=G_{0}^{\perp} \subset G_{1}^{\perp} \subset G_{2}^{\perp} \subset \ldots \subset G_{n}^{\perp} \subset \ldots, \quad \bigcup_{n=0}^{\infty} G_{n}^{\perp}=X .
$$


Фактор-группы $G_{n+1}^{\perp} \backslash G_{n}^{\perp}$ конечны и имеют порядок $p_{n}$. При каждом $n \in \mathbb{N}_{0}$ выберем элемент $g_{n} \in G_{n} \backslash G_{n+1}$ и зафиксируем. Каждый элемент $x \in G_{0}$ однозначно представим в виде

$$
x=\sum_{n=0}^{\infty} a_{n} g_{n}\left(a_{n}=\overline{0, p_{n}-1}\right),
$$

где сходимость понимается в смысле топологии, порожденной цепочной подгрупп (1). При каждом $n \in \mathbb{N}_{0}$ выберем характер $r_{n} \in G_{n+1}^{\perp} \backslash G_{n}^{\perp}$ и зафиксируем. Функции $r_{n}$ называются функциями Радемахера. Тогда характер $\chi \in X$ однозначно представим в виде

$$
\chi=\prod_{n=0}^{\infty}\left(r_{n}\right)^{\alpha_{n}}\left(\alpha_{n}=\overline{0, p_{n}-1}\right),
$$

причем в произведении (2) лишь конечное число $\alpha_{n}$ отлично от нуля, т. е.

$$
\chi=\prod_{n=0}^{M}\left(r_{n}\right)^{\alpha_{n}}
$$

число $M$ определяется характером $\chi$. В группе характеров можно ввести нумерацию следующим образом: если $\chi$ представлен в виде (3), то поставим ему в соответствие число

$$
n=\sum_{k=0}^{M} \alpha_{k} m_{k}
$$

и соответствующий характер будем обозначать через $\chi_{n}$.

Определим на группе $G$ систему функций Хаара следующим образом [7]. Положим $H_{0}(x) \equiv 1$, $H_{j m_{s}+k}=\sqrt{\left(m_{s}\right)} r_{s}^{j}(x \dot{-} q) 1_{G_{s}}(x \dot{-} q)$, где $j=\overline{1, p_{s}-1}, s=0,1, \ldots, k=\overline{0, m_{s}-1}, k$ и $q$ связаны соотношением $k=a_{0} m_{0}+a_{1} m_{1}+\ldots+a_{s-1} m_{s-1}, q=a_{0} g_{0} \dot{+} a_{1} g_{1} \dot{+} \ldots \dot{+} a_{s-1} g_{s-1}, a_{n}=\overline{0, p_{n}-1}$.

Определение 1 [3]. Пусть функция $f: G \rightarrow \mathbb{C}, T_{n}(\gamma, x)=\sum_{k=0}^{n-1} \gamma_{k} \chi_{k}(x), \gamma=\left(\gamma_{i}\right)_{i=0}^{\infty}, \gamma_{i} \in \mathbb{R}$. Если для функции $f \in L_{1}(G)$ существует функция $g: G \rightarrow \mathbb{C}$ из $L_{1}(G)$ такая, что

$$
\lim _{n \rightarrow \infty}\left\|\left(T_{n}(\gamma, \cdot) * f\right)(x)-g(x)\right\|_{L_{1}}=0,
$$

то $g$ называется сильной производной функции $f$ по системе характеров относительно последовательности $\left(\gamma_{i}\right)_{i=0}^{\infty}$ и обозначается $g=D_{\gamma}^{\chi} f$.

Определение 2. Пусть функция $f: G \rightarrow \mathbb{C}, \mathbf{K}_{\mathbf{n}}(\mathbf{f})=\int_{G} f(t) K_{n}(\gamma, x, t) d t, K_{n}(\gamma, x, t)=\gamma_{0}+$ $+\sum_{s=0}^{n} \sum_{j=1}^{p_{s}-1} \sum_{k=0}^{m_{s}-1} \gamma_{j m_{s}+k} H_{j m_{s}+k}(x) \bar{H}_{j m_{s}+k}(t), \gamma=\left(\gamma_{i}\right)_{i=0}^{\infty}, \gamma_{i} \in \mathbb{R}$. Если для функции $f \in L_{1}(G)$ существует функция $g: G \rightarrow \mathbb{C}$ из $L_{1}(G)$ такая, что

$$
\lim _{n \rightarrow \infty}\left\|\mathbf{K}_{\mathbf{n}}(\mathbf{f})-g(x)\right\|_{L_{1}}=0
$$

то $g$ называется сильной производной функции $f$ по системе Хаара относительно последовательности $\left(\gamma_{i}\right)_{i=0}^{\infty}$ и обозначается $g=D_{\gamma}^{H} f$.

Замечание 1. В определениях 1 и 2 оператор, который функции $f$ ставит в соответствие ее сильную производную $g$, обычно называют псевдодифференциальным опрератором, а последовательность $\gamma=\left(\gamma_{i}\right)_{i=0}^{\infty}-$ символом.

Замечание 2. Следует отметить, что в определении 1 (определении 2) на последовательность $\gamma=\left(\gamma_{i}\right)_{i=0}^{\infty}$ не наложено никаких ограничений, кроме $\gamma_{i} \in \mathbb{R}$, поэтому мы можем рассматривать последовательность следующего вида: $\left(\gamma_{i}^{l}\right)_{i=0}^{\infty}$, где $l$ означает показатель степени, $l \in \mathbb{Z}$. Если для такой последовательности условие (4) (условие (5)) выполнено, то в случае $l>0$ функцию $g: G \rightarrow \mathbb{C}$ будем называть сильной производной порядка $l$ функции $f$ по системе характеров (системе Хаара) относительно последовательности $\left(\gamma_{i}^{l}\right)_{i=0}^{\infty}$ и обозначать $g=D_{\gamma^{l}}^{\chi} f\left(g=D_{\gamma^{l}}^{H} f\right)$. А в случае $l<0$ функцию $g: G \rightarrow \mathbb{C}$ будем называть неопределенным сильным интегралом порядка $l$ функции $f$ по системе характеров (системе Хаара) относительно последовательности $\left(\gamma_{i}^{l}\right)_{i=0}^{\infty}$ и обозначать $g=D_{1 / \gamma^{l}}^{\chi} f$ $\left(g=D_{1 / \gamma^{l}}^{H} f\right)$. 
Более того, мы можем вместо последовательности $\left(\gamma_{i}\right)_{i=0}^{\infty}$ рассматривать последовательность $\left(1 / \gamma_{i}^{l}\right)_{i=0}^{\infty} l \in \mathbb{R} \backslash \mathbb{Z}$. В этом случае при $l>0$ функцию $g$ будем называть сильной производной дробного порядка $l$ функции $f$ по системе характеров (системе Хаара) относительно последовательности $\left(\gamma_{i}^{l}\right)_{i=0}^{\infty}$ и обозначать $g=D_{\gamma^{l}}^{\chi} f\left(g=D_{\gamma^{l}}^{H} f\right)$. В случае $l<0$ функцию $g$ будем называть неопределенным сильным интегралом дробного порядка $l$ функции $f$ по системе характеров (системе Хаара) относительно последовательности $\left(\gamma_{i}^{l}\right)_{i=0}^{\infty}$ и обозначать $g=D_{1 / \gamma^{l}}^{\chi} f\left(g=D_{1 / \gamma^{l}}^{H} f\right)$.

\section{2. ВСПОМОГАТЕЛЬНЫЕ РЕЗУЛЬТАТЫ}

Теорема 1. Пусть $f \in L_{1}(G)$ дифференцииуема в смысле определения 1 и пусть $g$ из $L_{1}(G)-$ ее сильная производная по системе характеров относительно последовательности $\gamma=\left(\gamma_{i}\right)_{i=0}^{\infty}$. Пусть $\sum_{k=0}^{\infty} c_{k} \chi_{k}$ - ряд Фурье функции $f$, тогда ряд Фурье функции $g$ имеет вид $\sum_{k=0}^{\infty} \gamma_{k} c_{k} \chi_{k}$, причем $g=\sum_{k=0}^{\infty} \gamma_{k} c_{k} \chi_{k}$

Доказательство. Рассмотрим свертку $\left(T_{l}(\gamma, \cdot) * f\right)(x)$ :

$$
\begin{gathered}
\left(T_{l}(\gamma, \cdot) * f\right)(x)=\int_{G} f(t) T_{l}(\gamma, x \dot{-} t) d \mu(t)=\sum_{k=0}^{l-1} \gamma_{k} \int_{G} f(t) \chi_{k}(x \dot{-} t) d \mu(t)= \\
=\sum_{k=0}^{l-1} \gamma_{k} \chi_{k}(x) \int_{G} f(t) \overline{\chi_{k}(t)} d \mu(t)=\sum_{k=0}^{l-1} \gamma_{k} c_{k} \chi_{k}(x) .
\end{gathered}
$$

Таким образом получаем, что ряд Фурье свертки $\left(T_{l}(\gamma, \cdot) * f\right)(x)$ сходится к самой свертке: $\sum_{k=0}^{l-1} \gamma_{k} c_{k} \chi_{k}=\left(T_{l}(\gamma, \cdot) * f\right)(x)$. Так как $g-$ сильная производная функции $f$ по системе характеров, то согласно определению 1 справедливо равенство (4). Следовательно, ряд Фурье функции $g$ имеет вид $\sum_{k=0}^{\infty} \gamma_{k} c_{k} \chi_{k}$ и $g=\sum_{k=0}^{\infty} \gamma_{k} c_{k} \chi_{k}$, сходимость пониматся в смысле нормы в $L_{1}$.

Следствие. Пусть $f \in L_{1}(G)$ и пусть ее ряд Фурье имеет вид: $\sum_{k=0}^{\infty} c_{k} \chi_{k}$. Если существует последовательность $\gamma=\left(\gamma_{i}\right)_{i=0}^{\infty}$, такая что функция $f$ дифференцируема в смьсле определения 1 , а ее сильная производная g интегрируема (см. замечание 2 к определениям 1, 2), то справедливо следующее равенство $\sum_{k=0}^{\infty} c_{k} \chi_{k}=f(x)$.

Теорема 2. Пусть $f \in L_{1}(G)$ дифференцируема в смьсле определения 2 и пусть $g$ из $L_{1}(G)-$ ее сильная производная по системе Хаара. Пусть $\sum_{k=0}^{\infty} d_{k} H_{k}-$ ряд Фурье функции $f$, тогда ряд Фурье функции g имеет вид $-\sum_{k=0}^{\infty} \gamma_{k} d_{k} H_{k}$, причем $g=\sum_{k=0}^{\infty} \gamma_{k} d_{k} H_{k}$.

Доказательство. Рассмотрим $\mathbf{K}_{\mathbf{n}}(\mathbf{f})$ :

$$
\begin{gathered}
\mathbf{K}_{\mathbf{n}}(\mathbf{f})=\int_{G} f(t)\left(\gamma_{0}+\sum_{s=0}^{n} \sum_{j=1}^{p_{s}-1} \sum_{k=0}^{m_{s}-1} \gamma_{j m_{s}+k} H_{j m_{s}+k}(x) \bar{H}_{j m_{s}+k}(t)\right) d \mu(t)= \\
=\gamma_{0} \int_{G} f(t) d \mu(t)+\sum_{s=0}^{n} \sum_{j=1}^{p_{s}-1} \sum_{k=0}^{m_{s}-1} \gamma_{j m_{s}+k} H_{j m_{s}+k}(x) \int_{G} f(t) \bar{H}_{j m_{s}+k}(t) d \mu(t)= \\
=\gamma_{0} d_{0}+\sum_{s=0}^{n} \sum_{j=1}^{p_{s}-1} \sum_{k=0}^{m_{s}-1} \gamma_{j m_{s}+k} d_{j m_{s}+k} H_{j m_{s}+k}(x) .
\end{gathered}
$$

Нетрудно убедится в том, что ряд Фурье функции $\mathbf{K}_{\mathbf{n}}(\mathbf{f})$ имеет вид

$$
\gamma_{0} d_{0}+\sum_{s=0}^{n} \sum_{j=1}^{p_{s}-1} \sum_{k=0}^{m_{s}-1} \gamma_{j m_{s}+k} d_{j m_{s}+k} H_{j m_{s}+k}(x),
$$


т. е. сходится к самой функции $\mathbf{K}_{\mathbf{n}}(\mathbf{f})$. Так как $g-$ сильная производная функции $f$ по системе Хаара, следовательно, справедливо равенство (5). Но тогда ряд Фурье функции $g$ имеет вид

$$
\gamma_{0} d_{0}+\sum_{s=0}^{\infty} \sum_{j=1}^{p_{s}-1} \sum_{k=0}^{m_{s}-1} \gamma_{j m_{s}+k} d_{j m_{s}+k} H_{j m_{s}+k}(x) \quad \text { и } \quad g=\gamma_{0} g_{0}+\sum_{s=0}^{\infty} \sum_{j=1}^{p_{s}-1} \sum_{k=0}^{m_{s}-1} \gamma_{j m_{s}+k} d_{j m_{s}+k} H_{j m_{s}+k}(x),
$$

сходимость понимается в смысле нормы в $L_{1}$.

Следствие. Пусть $f \in L_{1}(G)$ и ее ряд Фурье имеет вид $\sum_{k=0}^{\infty} c_{k} H_{k}$. Если существует последовательность $\gamma=\left(\gamma_{i}\right)_{i=0}^{\infty}$ такая, что функция $f$ дифференцируема в смьсле определения 2, а ее сильная производная g интегрируема (см. замечание $2 \kappa$ определениям 1, 2), то справедливо следующее равенство: $\sum_{k=0}^{\infty} c_{k} H_{k}=f(x)$.

\section{3. ОСНОВНЫЕ РЕЗУЛЬТАТЫ}

Теорема 3. Пусть функциия $f: G \rightarrow \mathbb{C}, f \in L_{1}(G)$. Пусть $f$ дифференцируема в смьсле определений 1 и 2 и $\sum_{k=0}^{\infty} c_{k} \chi_{k}$ - ряд Фурье функции $f$ по системе характеров, $\sum_{k=0}^{\infty} d_{k} H_{k}-$ ряд Фурье функции $f$ по системе Хаара. Тогда, если коэффициенты $\gamma_{k}$ удовлетворяют условиям:

$$
\gamma_{m_{n}}=\gamma_{m_{n}+1}=\ldots=\gamma_{m_{n+1}-1}, \quad n \in \mathbb{N}_{0},
$$

mo

$$
D_{\gamma}^{\chi} f=D_{\gamma}^{H} f
$$

где $D_{\gamma}^{\chi} f=\sum_{k=0}^{\infty} c_{k} \gamma_{k} \chi_{k}-$ ряд Фурье производной функции $f$ по системе характеров, $D_{\gamma}^{H} f=$ $=\sum_{k=0}^{\infty} d_{k} \gamma_{k} H_{k}-$ ряд Фурье производной функции $f$ по системе Хаара.

Доказательство. Рассмотрим частные суммы с номерами $m_{n}$ рядов Фурье по системе характеров и системе Хаара:

$$
S_{m_{n}}^{\chi}=\sum_{k=0}^{m_{n}-1} c_{k} \chi_{k}, \quad S_{m_{n}}^{H}=\sum_{k=0}^{m_{n}-1} d_{k} H_{k}
$$

Известно, что и система характеров, и система Хаара являются полными ортонормированными, следовательно, каждую функцию одной системы можно раскладывать в ряд Фурье по другой системе, т. е. если $k=\overline{0, m_{N}-1}$, то $\chi_{k}=\sum_{i=0}^{m_{N}-1} a_{k i} H_{i}$, причем в силу ортонормированности систем матрица $\left(a_{k i}\right)$ - ортогональная. Рассмотрим частную сумму с номером $m_{n}$ ряда Фурье по системе характеров:

$$
\begin{aligned}
& S_{m_{n}}^{\chi}=\sum_{k=0}^{m_{n}-1} c_{k} \chi_{k}=\sum_{k=0}^{m_{n}-1}\left(f, \chi_{k}\right) \chi_{k}=\sum_{k=0}^{m_{n}-1}\left(f, \sum_{i=0}^{m_{n}-1} a_{k i} H_{i}\right) \sum_{j=0}^{m_{n}-1} a_{k j} H_{j}= \\
= & \sum_{j=0}^{m_{n}-1} H_{j} \sum_{i=0}^{m_{n}-1}\left(f, H_{i}\right) \sum_{k=0}^{m_{n}-1} \overline{a_{k i}} a_{k j}=\sum_{j=0}^{m_{n}-1}\left(f, H_{j}\right) H_{j}=\sum_{j=0}^{m_{n}-1} d_{j} H_{j}=S_{m_{n}}^{H} .
\end{aligned}
$$

Таким образом, мы получили, что частные суммы с номерами $m_{n}$ рядов Фурье по системе характеров и системе Хаара совпадают. Рассмотрим теперь ряд Фурье производной функции $f$ по системе характеров:

$$
\begin{aligned}
& c_{0} \gamma_{0}+\sum_{n=1}^{\infty} \sum_{i=m_{n-1}}^{m_{n}-1} c_{i} \gamma_{i} \chi_{i}=c_{0} \gamma_{0}+\sum_{n=1}^{\infty} \gamma_{m_{n-1}} \sum_{i=m_{n-1}}^{m_{n}-1} c_{i} \chi_{i}= \\
= & \gamma_{0} d_{0}+\sum_{n=1}^{\infty} \gamma_{m_{n-1}} \sum_{i=m_{n-1}}^{m_{n}-1} d_{i} H_{i}=\gamma_{0} d_{0}+\sum_{n=1}^{\infty} \sum_{i=m_{n-1}}^{m_{n}-1} d_{i} \gamma_{i} H_{i} .
\end{aligned}
$$

Следовательно, используя теоремы 1 и 2 , мы получаем, что $D_{\gamma}^{\chi} f=D_{\gamma}^{H} f$. 


\section{4. МНОГОМЕРНЫЙ СЛУЧАЙ}

Пусть $(G, \dot{+})$ - компактная нуль-мерная группа, определенная в $\S 1$. Ограничимся рассмотрением случая, когда все $p_{n}=p$. Обозначим через $\mathbb{G}=G^{d}=G \times G \times \ldots \times G$ прямое произведение $d$ экземпляров группы $G$. Ясно, что элементами $\mathbb{G}$ являются $d$-мерные векторы, координаты которых - элементы группы $G$. Прямое произведение $d$ экземпляров подгруппы $G_{n}$ обозначим через $\mathbb{G}_{n d}=G_{n}^{d}=G_{n} \times G_{n} \times \ldots \times G_{n}$. Отметим, что данные подгруппы удовлетворяют свойству:

$$
\mathbb{G}=G_{0}^{d} \supset G_{1}^{d} \supset \ldots \supset G_{n}^{d} \supset \ldots \quad\left(\mathbb{G}=\mathbb{G}_{0} \supset \mathbb{G}_{d} \supset \ldots \supset \mathbb{G}_{n d} \supset \ldots\right),
$$

однако фактор-группы $\mathbb{G}_{n d} / \mathbb{G}_{(n+1) d}$ имеют порядок $p^{d}$, который не является простым числом. Поэтому такая цепочка не является основной. Пополним данную цепочку новыми подгруппами следующим образом $[5,6]$. Пусть $\mathbb{G}_{(n+1) d} \subset \mathbb{G}_{n d}$. Возьмем элемент $\bar{g}_{(n+1) d-1} \in \mathbb{G}_{n d} / \mathbb{G}_{(n+1) d}$, $\bar{g}_{(n+1) d-1}=\left(a_{1,1} g_{n}, a_{1,2} g_{n}, \ldots, a_{1, d} g_{n}\right)$, где $\left(g_{n}\right)-$ базисная последовательность в одномерной группе $G, a_{1, j}=\overline{0, p-1}$. Теперь образуем новую подгруппу $\mathbb{G}_{(n+1) d-1}$ следующим образом:

$$
\mathbb{G}_{(n+1) d-1}=\bigsqcup_{j=0}^{p-1} \mathbb{G}_{(n+1) d} \dot{+} j \bar{g}_{(n+1) d-1} .
$$

Таким образом, мы получили новую подгруппу, которая удовлетворяет следующим свойствам: $\mathbb{G}_{(n+1) d} \subset \mathbb{G}_{(n+1) d-1} \subset \mathbb{G}_{n d},\left(\mathbb{G}_{(n+1) d-1} / \mathbb{G}_{(n+1) d}\right)^{\sharp}=p$ и $\left(\mathbb{G}_{n d} / \mathbb{G}_{(n+1) d-1}\right)^{\sharp}=p^{d-1}$. Если $d>2$, продолжим наши построения. Выберем элемент $\bar{g}_{(n+1) d-2}=\left(a_{2,1} g_{n}, a_{2,2} g_{n}, \ldots, a_{2, d} g_{n}\right) \in \mathbb{G}_{n d} / \mathbb{G}_{(n+1) d-1}$, $a_{2, j}=\overline{0, p-1}$ таким образом, чтобы он был линейно независим с элементом $\bar{g}_{(n+1) d-1}$. С его помощью построим новую подгруппу:

$$
\mathbb{G}_{(n+1) d-2}=\bigsqcup_{j=0}^{p-1} \mathbb{G}_{(n+1) d} \dot{+j} \bar{g}_{(n+1) d-2}
$$

такую, что

$$
\begin{aligned}
\mathbb{G}_{(n+1) d} \subset \mathbb{G}_{(n+1) d-1} \subset \mathbb{G}_{(n+1) d-2} \subset \mathbb{G}_{n d}, \quad\left(\mathbb{G}_{(n+1) d-2} / \mathbb{G}_{(n+1) d-1}\right)^{\sharp}=p, \\
\left(\mathbb{G}_{n d} / \mathbb{G}_{(n+1) d-2}\right)^{\sharp}=p^{d-2} .
\end{aligned}
$$

Продолжая наши рассуждения, мы получим последовательность вложенных подгрупп, а именно

$$
\mathbb{G}_{n d} \supset \mathbb{G}_{(n+1) d-d+1} \supset \ldots \supset \mathbb{G}_{(n+1) d-1} \supset \mathbb{G}_{(n+1) d},
$$

удовлетворяющих условию $\left(\mathbb{G}_{(n+1) d-j} \backslash \mathbb{G}_{(n+1) d-j+1}\right)^{\sharp}=p$. Таким образом, мы получили, что для того чтобы пополнить цепочку в $d$-мерной группе $\mathbb{G}$, необходимо использовать невырожденную матрицу $A$. Матрица $A$ имеет вид

$$
\left(\begin{array}{cccc}
a_{1,1} & a_{1,2} & \ldots & a_{1, d} \\
a_{2,1} & a_{2,2} & \ldots & a_{2, d} \\
\vdots & \vdots & \ddots & \vdots \\
a_{d, 1} & a_{d, 2} & \ldots & a_{d, d}
\end{array}\right) .
$$

Следует отметить, что на каждом шаге $n$ мы используем одну и ту же матрицу $A$. Однако саму матрицу $A$ можно выбирать по-разному. От того какую мы матрицу выберем, зависит структура тех подгрупп, которыми мы пополняем цепочку подгрупп.

Теорема 4. Пусть $\mathbb{G}$ есть d-мерная группа. Обозначим:

$$
\begin{gathered}
M=\left\{\chi_{\bar{\alpha}}(\bar{x})=\chi_{\alpha_{1}}\left(x^{1}\right) \chi_{\alpha_{2}}\left(x^{2}\right) \ldots \chi_{\alpha_{d}}\left(x^{d}\right), \quad \bar{\alpha}=\left(\alpha_{1}, \alpha_{2}, \ldots, \alpha_{d}\right), \quad \alpha_{j}=\overline{0, m_{n+1}-1}\right. \\
\chi_{\alpha_{1}}\left(a_{1,1} g_{n}\right) \chi_{\alpha_{2}}\left(a_{1,2} g_{n}\right) \ldots \chi_{\alpha_{d}}\left(a_{1, d} g_{n}\right)=1, \quad \chi_{\alpha_{1}}\left(a_{2,1} g_{n}\right) \chi_{\alpha_{2}}\left(a_{2,2} g_{n}\right) \ldots \chi_{\alpha_{d}}\left(a_{2, d} g_{n}\right)=1, \ldots \\
\left.\chi_{\alpha_{1}}\left(a_{k, 1} g_{n}\right) \chi_{\alpha_{2}}\left(a_{k, 2} g_{n}\right) \ldots \chi_{\alpha_{d}}\left(a_{k, d} g_{n}\right)=1, \quad \chi_{\alpha_{j}} \in G_{n+1}^{\perp}\right\}
\end{gathered}
$$

mогда $\mathbb{G}_{(n+1) d-k}^{\perp}=M$.

Доказательство. Для того чтобы доказать равенство двух множеств, докажем два включения. Покажем, что $M \subset \mathbb{G}_{(n+1) d-k}$. Рассмотрим произвольный элемент $\bar{x} \in \mathbb{G}_{(n+1) d-k}: \bar{x}=\bar{x}_{k-1} \dot{+}$ $\dot{+} j_{k-1}\left(a_{k, 1} g_{n}, \ldots, a_{k, d} g_{n}\right)$, где $\bar{x}_{k-1} \in \mathbb{G}_{(n+1) d-k+1}, j_{k-1}=\overline{0, p-1}$. В свою очередь, элемент $\bar{x}_{k-1}=$ 
$=\bar{x}_{k-2} \dot{+} j_{k-2}\left(a_{k-1,1} g_{n}, \ldots, a_{k-1, d} g_{n}\right)$, где $\bar{x}_{k-2} \in \mathbb{G}_{(n+1) d-k+2}, j_{k-2}=\overline{0, p-1}$. Продолжая наши рассуждения мы дойдем до элемента $\bar{x}_{1} \in \mathbb{G}_{(n+1) d-1}$, который можно представить в виде $\bar{x}_{1}=\bar{x}_{0}+j_{0}\left(a_{1,1} g_{n}, \ldots, a_{1, d} g_{n}\right)$, где $\bar{x}_{0} \in \mathbb{G}_{(n+1) d}, j_{0}=\overline{0, p-1}$. Таким образом, мы получили следующее представление элемента:

$$
\bar{x}=\bar{x}_{0} \dot{+} j_{0}\left(a_{1,1} g_{n}, \ldots, a_{1, d} g_{n}\right) \dot{+} \ldots \dot{+} j_{k-2}\left(a_{k-1,1} g_{n}, \ldots, a_{k-1, d} g_{n}\right) \dot{+} j_{k-1}\left(a_{k, 1} g_{n}, \ldots, a_{k, d} g_{n}\right) .
$$

Теперь выберем произвольный элемент $\chi_{\bar{\alpha}} \in M$, зафиксируем и рассмотрим его значение на элементе $\bar{x}$ :

$$
\begin{gathered}
\chi_{\bar{\alpha}}(\bar{x})=\chi_{\bar{\alpha}}\left(\bar{x}_{0}\right) \chi_{\bar{\alpha}}\left(j_{0}\left(a_{1,1} g_{n}, \ldots, a_{1, d} g_{n}\right)\right) \times \ldots \\
\ldots \times \chi_{\bar{\alpha}}\left(j_{k-1}\left(a_{k-1,1} g_{n}, \ldots, a_{k-1, d} g_{n}\right)\right) \chi_{\bar{\alpha}}\left(j_{k}\left(a_{k, 1} g_{n}, \ldots, a_{k, d} g_{n}\right)\right) .
\end{gathered}
$$

Первый множитель $\chi_{\bar{\alpha}}\left(\bar{x}_{0}\right)=1$, так как $\bar{x}_{0} \in \mathbb{G}_{(n+1) d}$, т. е. $x_{0}^{(j)} \in G_{n+1}$ и $\chi_{\alpha_{j}} \in G_{n+1}^{\perp}$. Остальные множители также равны единице, поскольку это прямо следует из определения множества $M$. Таким образом, мы установили, что для любого $\bar{x} \in \mathbb{G}_{(n+1) d-k} \chi_{\bar{\alpha}}(\bar{x})=1$, следовательно, $\chi_{\bar{\alpha}} \in \mathbb{G}_{(n+1) d-k}^{\perp}$. А так как $\chi \bar{\alpha}$ произвольный, следовательно, $M \subset \mathbb{G}_{(n+1) d-k}$.

Прежде чем показать обратное включение, определим вид характеров в многомерной группе. Рассмотрим произвольный характер $\chi \in \mathbb{G}_{(n+1) d}^{\perp}$. Рассмотрим элемент $\bar{x} \in \mathbb{G}_{(n+1) d}$, его можно представить в виде $\bar{x}=\left(x^{(1)}, x^{(2)}, \ldots, x^{(d)}\right)=\left(x^{(1)}, 0, \ldots, 0\right) \dot{+}\left(0, x^{(2)}, \ldots, 0\right) \dot{+}\left(0,0 \ldots, x^{(d)}\right)$. При таком разложении каждый из сомножителей можно рассматривать как одномерный элемент из $G_{n+1}$. Рассмотрим значение характера на этом элементе $\chi(\bar{x})=\chi\left(\left(x^{(1)}, 0, \ldots, 0\right)\right) \chi\left(\left(0, x^{(2)}, \ldots, 0\right) \ldots \chi\left(\left(0,0, \ldots, x^{(d)}\right)\right)\right.$. Поскольку в правой части равенства участвуют вектора, которые мы обусловились считать одномерными, то вместо $\chi$ можно рассмотреть одномерные характеры $\chi_{\alpha_{j}}$, т. е. записать: $\chi(\bar{x})=\chi_{\alpha_{1}}\left(x^{(1)}\right) \chi_{\alpha_{2}}\left(x^{(2)}\right) \ldots \chi_{\alpha_{d}}\left(x^{(d)}\right)$. Проверим, сохранятся ли при этом все свойства характера $\chi$. Используя свойства одномерных характеров, можно сразу получить, что при таком представлении $|\chi(\bar{x})|=1$ и $\chi(\bar{x} \dot{+} \bar{y})=\chi(\bar{x}) \chi(\bar{y})$. Рассмотрим элемент $\bar{x} \in \mathbb{G}_{(n+1) d}$, следовательно, по определению аннулятора $\chi(\bar{x})=1$. Так как $\chi \in \mathbb{G}_{(n+1) d}^{\perp}$, следовательно, на любом элементе из $\mathbb{G}_{(n+1) d}$ значение характера должно быть равным единице. Рассмотрим $\bar{x} \in \mathbb{G}_{(n+1) d}$, его координаты $-x^{(j)} \in G_{n+1}$. Для того чтобы сохранилось свойство $\chi(\bar{x})=1$, необходимо, чтобы наши одномерные характеры, участвующие в представлении многомерного характера, были из аннулятора $G_{n+1}^{\perp}$. Таким образом, мы получили, что любой многомерный характер можно представить в виде произведения одномерных. Докажем теперь, что $\mathbb{G}_{(n+1) d-k} \subset M$. Пусть $\chi \in \mathbb{G}_{(n+1) d-k}$. Следовательно, по определению аннулятора на любом элементе $\bar{x} \in \mathbb{G}_{(n+1) d-k} \chi(\bar{x})=1$. Рассмотрим $\chi\left(a_{1,1} g_{n}, a_{1,2} g_{n}, \ldots, a_{1, d} g_{n}\right)=\chi\left(\bar{g}_{(n+1) d-1}\right)=1$, так как $\bar{g}_{(n+1) d-1} \in \mathbb{G}_{(n+1) d-1} \subset \mathbb{G}_{(n+1) d-k} \cdot \chi\left(a_{2,1} g_{n}, a_{2,2} g_{n}, \ldots, a_{2, d} g_{n}\right)=\chi\left(\bar{g}_{(n+1) d-2}\right)=1$, так как $\bar{g}_{(n+1) d-2} \in \mathbb{G}_{(n+1) d-2} \subset \mathbb{G}_{(n+1) d-k} \cdot \chi\left(a_{k, 1} g_{n}, a_{k, 2} g_{n}, \ldots, a_{k, d} g_{n}\right)=\chi\left(\bar{g}_{(n+1) d-k}\right)=1$, так как $\bar{g}_{(n+1) d-k} \in \mathbb{G}_{(n+1) d-k} \subset \mathbb{G}_{(n+1) d-k}$. Таким образом, получили, что $\chi \in M$, т. е. $\mathbb{G}_{(n+1) d-k} \subset M$. Мы доказали два включения, следовательно, $\mathbb{G}_{(n+1) d-k}=M$.

Замечание. Аннуляторы $\mathbb{G}_{n d}^{\perp}$ имеют более простой вид, а именно

$$
\mathbb{G}_{n d}^{\perp}=\left\{\chi_{\bar{\alpha}}(\bar{x})=\chi_{\alpha_{1}}\left(x^{(1)}\right) \chi_{\alpha_{2}}\left(x^{(2)}\right) \ldots \chi_{\alpha_{d}}\left(x^{(d)}\right), \chi_{\alpha_{j}} \in G_{n}^{\perp}\right\} .
$$

После того как в $\mathbb{G}=G^{d}$ мы пополнили цепочку подгрупп до основной

$$
\mathbb{G}_{n d} \supset \mathbb{G}_{(n+1) d-d+1} \supset \ldots \supset \mathbb{G}_{(n+1) d-1} \supset \mathbb{G}_{(n+1) d}
$$

и определили вид аннуляторов группы $\mathbb{G}$, мы можем рассматривать нашу многомерную группу $\mathbb{G}$ как одномерную. Используя при пополнении цепочки подгрупп разные матрицы, мы получим различные группы $G$. Если на таких различных группах, образованных из одной многомерной, определять производные в смысле определений 1 и 2, то на каждой из образованных группах эти производные будут отличаться. Однако существует способ определения производной таким образом, чтобы она не зависела от матрицы, с помощью которой образуются дополнительные подгруппы.

Определение 3. Пусть функция $f: \mathbb{G} \rightarrow \mathbb{C}, f \in L_{1}(G)$,

$$
T_{l}(\gamma, \bar{x})=\sum_{n=0}^{l-1} \sum_{\substack { \bar{k}: \\
\begin{subarray}{c}{k_{j}=\overline{0, m_{n+1}-1} \\
\max _{j}{ \overline { k } : \\
\begin{subarray} { c } { k _ { j } = \overline { 0 , m _ { n + 1 } - 1 } \\
\operatorname { m a x } _ { j } } }\end{subarray}} \gamma_{\bar{k}} \chi_{\bar{k}}(\bar{x}),
$$


$\gamma=\left(\gamma_{\bar{i}}\right), \bar{i}=\left(i_{1}, i_{2}, \ldots, i_{d}\right), i_{j} \in \mathbb{N}_{0}, \gamma_{\bar{i}} \in \mathbb{R}$. Если для функции $f \in L_{1}(G)$ существует функция $g: \mathbb{G} \rightarrow \mathbb{C}$ из $L_{1}(G)$ такая, что

$$
\lim _{l \rightarrow \infty}\left\|\left(T_{l}(\gamma, \cdot) * f\right)(\bar{x})-g(\bar{x})\right\|_{L_{1}}=0,
$$

то $g$ называется сильной производной функции $f$ по системе характеров относительно последовательности $\left(\gamma_{\bar{i}}\right)$ и обозначается $g=D_{\bar{\gamma}}^{\bar{\chi}} f$.

Покажем теперь, что, используя определение 3 в многомерной группе, мы получим производные, которые не зависят от способа пополнения основной цепочки подгрупп (т. е. от матрицы $A$ ).

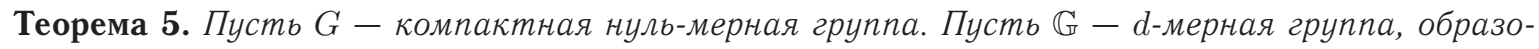
ванная прямым произведением d копий группь $G$. Цепочку подгрупп $\mathbb{G}_{0} \supset \mathbb{G}_{d} \supset \ldots \supset \mathbb{G}_{n d} \supset \ldots$ будем пополнять разными способами с помощью матрии $A_{1}$ и $A_{2}$. Пусть $f: \mathbb{G} \rightarrow \mathbb{C}$, пусть $g_{1}, g_{2}$ - сильные производные в смысле определения 3 , построенные по разным матрицам $A_{1}$ и $A_{2}$ соответственно. Если последовательность $\left(\gamma_{\bar{k}}\right)$ в определении 3 выбрать таким образом, чтобы $\gamma_{\bar{k}}$ принимали постоянное значение при $\bar{k}=\left(k_{1}, k_{2}, \ldots, k_{d}\right)$ таких, ито их координаты $\left(k_{j}\right)=\overline{0, m_{n+1}-1}, \max _{j} k_{j} \geq m_{n}$, тогда $g_{1}=g_{2}$.

Доказательство. Используя определение 3 и доказательство, аналогичное теоремам 1 и 2 , мы можем установить, что

$$
g_{1}=\sum_{n=0}^{\infty} \sum_{\substack{\bar{k}: k_{j}=\overline{0, m_{n+1}-1}, \max _{j} k_{j} \geq m_{n}}} \gamma_{\bar{k}} \chi_{\bar{k}}(\bar{x}), \quad g_{2}=\sum_{n=0}^{\infty} \sum_{\substack { \bar{l}: \\
\begin{subarray}{c}{l_{j} \\
\max _{j} \bar{p}_{j} \geq m_{n}+1{ \overline { l } : \\
\begin{subarray} { c } { l _ { j } \\
\operatorname { m a x } _ { j } \overline { p } _ { j } \geq m _ { n } + 1 } }\end{subarray}} \gamma_{\bar{l}} \chi_{\bar{l}}(\bar{x}) .
$$

Обратимся теперь к теореме 4 , в которой был показан вид аннуляторов $\mathbb{G}_{(n+1) d-k}^{\perp}$. Рассмотрим сначала аннулятор вида $\mathbb{G}_{n d}^{\perp}=\left\{\chi_{\bar{\alpha}}(\bar{x})=\chi_{\alpha_{1}}\left(x^{1}\right) \chi_{\alpha_{2}}\left(x^{2}\right) \ldots \chi_{\alpha_{d}}\left(x^{d}\right), \chi_{\alpha_{j}} \in G_{n+1}^{\perp}\right\}$ и определим, какие характеры (с какими векторными номерами) попали в этот аннулятор. Для одномерных групп известно, что $G_{n}^{\perp}=\left\{\chi_{k}, k=\overline{0, m_{n}-1}\right\}$. Следовательно, $\mathbb{G}_{n d}^{\perp}=\left\{\chi_{\bar{\alpha}}(\bar{x}), \bar{\alpha}=\left(\alpha_{1}, \alpha_{2}, \ldots, \alpha_{d}\right), \alpha_{j}=\overline{0, m_{n}-1}\right\}$. Рассмотрим теперь аннуляторы для подгрупп, которые мы получаем в результате пополнения цепочки до основной. Из теоремы 4 следует, что в аннулятор $\mathbb{G}_{(n+1) d-k}^{\perp}$ попадают не все характеры с номерами $\bar{\alpha}: \alpha_{j}=\overline{0, m_{n+1}-1}$, а какие-то из них, которые удовлетворяют условиям $\chi_{\alpha_{1}}\left(a_{1,1} g_{n}\right) \times$ $\times \chi_{\alpha_{2}}\left(a_{1,2} g_{n}\right) \ldots \chi_{\alpha_{d}}\left(a_{1, d} g_{n}\right)=1, \quad \chi_{\alpha_{2}}\left(a_{2,1} g_{n}\right) \chi_{\alpha_{2}}\left(a_{2,2} g_{n}\right) \ldots \chi_{\alpha_{d}}\left(a_{2, d} g_{n}\right)=1, \ldots, \quad \chi_{\alpha_{k}}\left(a_{k, 1} g_{n}\right) \times$ $\times \chi_{\alpha_{2}}\left(a_{k, 2} g_{n}\right) \ldots \chi_{\alpha_{d}}\left(a_{k, d} g_{n}\right)=1$. Если мы строим пополнения по различным матрицам, то соответственно эти условия также различны, поскольку определяются матрицами. Следовательно, аннуляторы $\mathbb{G}_{(n+1) d-k}^{\perp}$, построенные по разным матрицам, отличаются друг от друга набором характеров, которые в них попали. Однако если мы рассматриваем аннуляторы с номером $n d$, то при различных пополнениях, туда попадает один и тот же набор характеров. Соответственно если мы будем рассматривать пачки

$$
\sum_{\substack { \bar{k}: \\
\begin{subarray}{c}{k_{j}=0, m_{n+1}-1 \\
\max _{j} k_{j} \geq m_{n}{ \overline { k } : \\
\begin{subarray} { c } { k _ { j } = 0 , m _ { n + 1 } - 1 \\
\operatorname { m a x } _ { j } k _ { j } \geq m _ { n } } }\end{subarray}} \gamma_{\bar{k}} \chi_{\bar{k}}(\bar{x}),
$$

в которых $\gamma_{\bar{k}}=$ const, то такие суммы одинаковые. Отсюда несложно показать, что $g_{1}=g_{2}$.

Работа выполнена при финансовой поддержке РФФИ (проект № 13-01-00102).

\section{Библиографический список}

1. Butzer P. L. Wagner H. J. Walsh-Fourier series and the concept of a derivative // Appl. Anal. 1973. Vol. 3, № 1. P. 29-46.

2. Голубов Б. И. О модифицированном сильном двоичном интеграле и производной // Мат. сб. 2002. Т. 193, № 4. С. $37-60$.

3. Волосивец С. С. Модифицированный $\mathbf{P}$-ичный интеграл и модифицированная $\mathbf{P}$-ичная производная для функций, определенных на полуоси // Изв. вузов. Математика. 2005. № 6. С. 28-39.

4. Козырев С. В. Теория всплесков как $p$-адический спектральный анализ // Изв. РАН. Сер. математическая. 2002. Т. 66, № 2. С. 149-158.

5. Lukomskii S. F. Haar system on a product of zerodimensional compact group // Centr. Eur. J. Math. 2011. Vol. 9, № 3. P. 627-639.

6. Lukomskii S. F. Multiresolution analysis on product of 
мерной группе // Изв. Сарат. ун-та. Нов. сер. Сер. Математика. Механика. Информатика. 2009. Т. 9, вып. 1. C. 14-19.
2012. Vol. 385. P. 1162-1178.

7. Лукомский С. Ф. О рядах Хаара на компактной нуль-

\section{On Differential Operator in Compact Zero-dimensional Groups}

\section{S. Kruss}

Saratov State University, 83, Astrakhanskaya str., Saratov, 410012, Russia, KrussUS@gmail.com

We define strong derivative on zero-dimensional compact group and find conditions under which the differential operator does not depend from an orthonormal system that defines this derivative. For multidimensional case we find conditions under which the differential operator does not depend from method of conversion multidimensional group in one-dimensional group. We obtain a clear view of annihilators in a multidimensional compact zero-dimensional group.

Key words: zero-dimensional groups, pseudo-differential operator, strong $\mathbf{P}$-adic derivative, strong $\mathbf{P}$-adic integral.

This work was supported by the Russian Foundation for Basic Research (project no. 13-01-00102).

\section{References}

1. Butzer P. L. Wagner H. J. Walsh - Fourier series and the concept of a derivative. Appl.Anal, 1973, vol. 3. no. 1, pp. 29-46.

2. Golubov B. I. A modified strong dyadic integral and derivative. Sbornik: Mathematics, 2002, vol. 193, no. 4, pp. 507-529. DOI: 10.4213/sm643.

3. Volosivets S. S. A modified $P$-adic integral and a modified $P$-adic derivative for functions defined on a halfaxis. Russian Math. [Izvestiya VUZ. Matematika], 2005. vol. 49, no. 6. pp. 25-36.

4. Kozyrev S. V. Wavelet theory as $p$-adic spectral

analysis. Izv. Math., 2002, vol. 66, no. 2, pp. 367-376. DOI: $10.4213 /$ im 381 .

5. Lukomskii S. F. Haar system on a product of zerodimensional compact group. Centr. Eur. J. Math., 2011, vol. 9, no. 3, pp. 627-639.

6. Lukomskii S. F. Multiresolution analysis on product of zero-dimensional Abelian groups. J. Math. Anal. Appl., 2012, vol. 385, pp. 1162-1178.

7. Lukomskii S. F. Haar series on compact zerodimmesional abelian group. Izv. Saratov Univ. (N.S.), Ser. Math. Mech. Inform., 2009, vol. 9, no. 1, pp. 14-19 (in Russian).

УДК 517.51

\section{ПРИБЛИЖЕНИЕ ФУНКЦИЙ В СИММЕТРИЧНЫХ И СВЯЗАННЫХ С НИМИ ГЕЛЬДЕРОВЫХ ПРОСТРАНСТВАХ ЛИНЕЙНЫМИ СРЕДНИМИ РЯДОВ ФУРЬЕ}

\section{Т. В. Лихачева}

Аспирантка кафредры теории фрункций и приближений, Саратовский государственный университет им. Н. Г. Чернышевского, lofinaT@mail.ru

В статье некоторые методы суммирования применяются к рядам Фурье-Виленкина в так называемых симметричных пространствах. Эти методы используют треугольные матрицы, суммы по строкам которых стремятся к нулю, с некоторыми ограничениями на разности коэфрфициентов. Тригонометрические аналоги наших результатов принадлежат М. Л. Митталу, Б. Э. Родесу, А. Гувену и др.

Ключевые слова: система Виленкина, линейные средние, пространство Гельдера, симметричное (перестановочно-инвариантное) пространство.

\section{1. ОСНОВНЫЕ ПОНЯТИЯ}

Пусть $\mathbf{P}=\left\{p_{n}\right\}_{n=1}^{\infty}-$ последовательность натуральных чисел такая, что $2 \leq p_{n} \leq N$ при $n \in \mathbb{N}$. Положим по определению $m_{0}=1, m_{n}=p_{1} \cdots p_{n}$ при $n \in \mathbb{N} ; \mathbb{Z}\left(p_{n}\right)=\left\{0,1, \cdots, p_{n}-1\right\}$. Каждое число $x \in[0,1)$ может быть представлено в виде

$$
x=\sum_{n=1}^{\infty} x_{n} / m_{n}, \quad x_{n} \in \mathbb{Z}\left(p_{n}\right) .
$$

